\title{
JORDAN SUPERDERIVATIONS. II
}

\author{
MAJA FOŠNER
}

Received 16 April 2003

\begin{abstract}
In a recent paper we have extended the classical Herstein's theorem on Jordan derivations on prime rings to Jordan superderivations on prime associative superalgebras. In the present paper we extend this result to semiprime associative superalgebras.
\end{abstract}

2000 Mathematics Subject Classification: 16W25, 16W55, 17C50, 17C70.

1. Introduction. Throughout the paper, by an algebra we will mean an algebra over a fixed unital commutative ring $\Phi$, and we assume that $\Phi$ contains the element $1 / 2$.

Let $\mathscr{A}$ be an associative superalgebra, that is, a $\mathbb{Z}_{2}$-graded associative algebra. This means that there exist $\Phi$-submodules $\mathscr{A}_{0}, \mathscr{A}_{1}$ of $\mathscr{A}$ such that $\mathscr{A}=\mathscr{A}_{0} \oplus \mathscr{A}_{1}, \mathscr{A}_{0} \mathscr{A}_{0} \subseteq \mathscr{A}_{0}\left(\mathscr{A}_{0}\right.$ is a subalgebra of $\mathscr{A}), \mathscr{A}_{0} \mathscr{A}_{1} \subseteq \mathscr{A}_{1}, \mathscr{A}_{1} \mathscr{A}_{0} \subseteq \mathscr{A}_{1}\left(\mathscr{A}_{1}\right.$ is an $\mathscr{A}_{0}$-bimodule), and $\mathscr{A}_{1} \mathscr{A}_{1} \subseteq \mathscr{A}_{0}$. We say that $\mathscr{A}_{0}$ is the even and $\mathscr{A}_{1}$ is the odd part of $\mathscr{A}$. An element $a \in \mathscr{A}_{i}, i=0$ or $i=1$, is said to be homogeneous of degree $i$, and in this case we write $|a|=i$. An ideal $U$ of $\mathscr{A}$ is said to be graded if $U=U \cap \mathscr{A}_{0} \oplus U \cap \mathscr{A}_{1}$. A superalgebra $\mathscr{A}$ is called prime if the product of any two nonzero graded ideals in $\mathscr{A}$ is nonzero, and is called semiprime if it does not contain nonzero nilpotent graded ideals.

Introducing a new product in $\mathscr{A}$ by $x \circ_{s} y=(1 / 2)\left(x y+(-1)^{|x||y|} y x\right), x, y \in \mathscr{A}_{0} \cup \mathscr{A}_{1}$, $\mathscr{A}$ becomes a Jordan superalgebra. Over the recent years there has been a considerable interest in the relation between Jordan, Lie, and associative structures in associative superalgebras. The present paper continues this line of investigations. Some more details about the background of this research and a more comprehensive list of references are given in our preceding paper [6].

Let $i=0$ or $i=1$. A $\Phi$-linear map $D_{i}: \mathscr{A} \rightarrow \mathscr{A}$ such that $D\left(\mathscr{A}_{j}\right) \subseteq \mathscr{A}_{j+i}, i, j \in \mathbb{Z}_{2}$, is called a superderivation of degree $i$ if it satisfies

$$
D_{i}(x y)=D_{i}(x) y+(-1)^{i|x|} x D_{i}(y) \quad \forall x, y \in \mathscr{A}_{0} \cup \mathscr{A}_{1},
$$

and is called a Jordan superderivation of degree $i$ if it satisfies

$$
D_{i}\left(x \circ_{s} y\right)=D_{i}(x) \circ_{s} y+(-1)^{i|x|} x \circ_{s} D_{i}(y) \quad \forall x, y \in \mathscr{A}_{0} \cup \mathscr{A}_{1}
$$

A (general) superderivation is the sum of a superderivation of degree 0 and a superderivation of degree 1. Similarly, a Jordan superderivation is defined as the sum of Jordan superderivations of degrees 0 and 1. Superderivations are obviously Jordan superderivations, while the converse may not be true. In [6] we proved that every Jordan superderivation on a prime associative superalgebra $\mathscr{A}$ is a superderivation, unless $\mathscr{A}_{0}$ 
is a commutative algebra. The case when $\mathscr{A}_{0}$ is commutative is indeed exceptional, as shown by examples in [6].

The concept of a Jordan superderivation can be viewed as a generalization of the concept of a Jordan derivation of an associative algebra. Namely, in the case of trivial superalgebras (i.e., the odd part is 0), these two notions coincide. Herstein's classical result [7] from 1957 implies that every Jordan derivation on a prime algebra over $\Phi$ is a derivation. In 1975, Cusack [5] proved that the same result holds true in semiprime algebras (see also [3]). It is therefore natural to ask whether our result from [6] can be extended to semiprime superalgebras. Our main goal is to prove the following generalization.

THEOREM 1.1. Let $\mathscr{A}=\mathscr{A}_{0} \oplus \mathscr{A}_{1}$ be a semiprime associative superalgebra and let $D=$ $D_{0}+D_{1}$ be a Jordan superderivation. Then there exist graded ideals $U$ and $V$ of $A$ such that $D_{i}(u x)=D_{i}(u) x+(-1)^{i|u|} u D_{i}(x), i=0,1$, for all $u \in U$ and $x \in \mathscr{A}$, and $\left[v_{0}, x_{0}\right]=$ 0 for all $v_{0} \in V_{0}$ and $x_{0} \in A_{0}$. Moreover, $U \cap V=0$ and $U \oplus V$ is an essential ideal of $A$. If $U=0$, then $A_{0}$ is commutative, and if $V=0$, then $D$ is a superderivation.

In particular, this theorem shows that the restriction of $D$ to $U$ is a superderivation and the superalgebra $V$ has a commutative even part. The next example (a modification of the one given in [1, page 458]) shows that, in general, $U$ and $V$ cannot be chosen so that their sum is equal to $\mathscr{A}$.

EXAMPLE 1.2. Let $A=A_{0} \oplus A_{1}$ and $B=B_{0} \oplus B_{1}$ be prime associative superalgebras satisfying the following conditions: none of them contains an identity element, $A_{0}$ is a noncommutative algebra, $B$ is commutative (as an algebra) and $B_{1} \neq 0$. For example, one can take the trivial superalgebra of all finite-rank operators on an infinite-dimensional vector space (over a field $\Phi$ ) for $A$, and $B=X \Phi[X]$ (i.e., the algebra of polynomials over $\Phi$ with constant term 0 ) with graduation $B_{0}=\Phi\left[X^{2}\right]$ and $B_{1}=X \Phi\left[X^{2}\right]$. Let $\mathscr{A}=A \oplus B \oplus \Phi$ be the unitization of the algebra $A \oplus B$. Set $\mathscr{A}_{0}=A_{0} \oplus B_{0} \oplus \Phi 1$ and $\mathscr{A}_{1}=A_{1} \oplus B_{1}$ and note that thereby $\mathscr{A}$ becomes a semiprime associative superalgebra whose even part is noncommutative. Let $b_{0}+b_{1}=b \in B$ be such that $b_{0} \neq 0$ and define $D: A \rightarrow A$ by $D\left(x_{0}+x_{1}+y_{0}+y_{1}+\lambda\right)=b y_{1}$ for all $x_{0} \in A_{0}, x_{1} \in A_{1}, y_{0} \in B_{0}, y_{1} \in B_{1}$, and $\lambda \in \Phi$. Then $D$ is a Jordan superderivation which is not a superderivation. Since $\mathscr{A}$ is a unital algebra whose only central idempotents are 0 and $1, \mathscr{A}$ does not contain proper ideals $U$ and $V$ such that $\mathscr{A}=U \oplus V$.

In the proof of Theorem 1.1 we will, on the one hand, use several computations from [6], and, on the other hand, we will use some ideas from [2, 3, 4].

2. Preliminaries. We first fix the notation. Throughout the paper, by $\mathscr{A}=\mathscr{A}_{0} \oplus \mathscr{A}_{1}$ we will denote a semiprime associative superalgebra. It is easy to see that $\mathscr{A}$ is also semiprime as an algebra (i.e., if $a \in \mathscr{A}$ and $a \mathscr{A} a=0$, then $a=0$ ) and also $\mathscr{A}_{0}$ is a semiprime algebra [9, Lemma 1.2]. As usual, we will write $[a, b]=a b-b a$ and $a$ 。 $b=(1 / 2)(a b+b a)$ for $a, b \in \mathscr{A}$. By $Z(\mathscr{A})$ (resp., $Z\left(\mathscr{A}_{0}\right)$ ) we denote the center of $\mathscr{A}$ (resp., $\mathscr{A}_{0}$ ). Further, $D=D_{0}+D_{1}$ will denote a Jordan superderivation of $\mathscr{A}$, where, of course, $D_{i}, i=0,1$, denotes a Jordan superderivation of degree $i$. Define a bilinear map 
$\delta_{i}: \mathscr{A} \times \mathscr{A} \rightarrow \mathscr{A}$ by

$$
\delta_{i}(x, y)=D_{i}(x y)-D_{i}(x) y-(-1)^{i|x|} x D_{i}(y)
$$

for all $x, y \in \mathscr{A}_{0} \cup \mathscr{A}_{1}$. Clearly, $\delta_{i}=0$ if and only if $D_{i}$ is a superderivation of degree $i$.

If $x$ and $y$ are elements in $\mathscr{A}$ such that $x \mathscr{A} y=0$, then it follows that $x y=y x=$ $y \mathscr{A} x=0$. Namely, we have $y a(x \mathscr{A} y) a x=0$ for all $a \in \mathscr{A}$, which in turn implies $y \mathscr{A} x=$ 0 by the semiprimeness of $\mathscr{A}$. Similarly, from $x(y \mathscr{A} x) y=0$ and $y(x \mathscr{A} y) x=0$ we get $x y=y x=0$. For such elements $x, y$, we will write $x \perp y$.

The proof of Theorem 1.1 consists of several steps. First, we gather some auxiliary results which will be needed later.

LEMmA 2.1. Suppose that $a \in \mathscr{A}_{1}$ is such that ax $x_{1}, x_{1} a \in Z\left(\mathscr{A}_{0}\right)$ for all $x_{1} \in \mathscr{A}_{1}$. Then $a^{2} \in Z(\mathscr{A})$.

Proof. We have $0=\left[a^{2}, a x_{1}\right]=a\left[a^{2}, x_{1}\right]$ and $0=\left[a^{2}, x_{1} a\right]=\left[a^{2}, x_{1}\right] a$ for all $x_{1} \in \mathscr{A}_{1}$. Therefore $\left[a^{2},\left[a^{2}, \mathscr{A}_{1}\right]\right]=0$, which, together with $\left[a^{2},\left[a^{2}, \mathscr{A}_{0}\right]\right]=0$, gives $\left[a^{2},\left[a^{2}, \mathscr{A}\right]\right]=0$. But then $a^{2} \in Z(\mathscr{A})$ by $[8$, Lemma 1.1.9].

The next lemma is a slight extension of [6, Lemma 2.3].

LEMMA 2.2. Let $U$ be a graded ideal of $A$.

(i) If $u_{1} U_{1} u_{1}=0$, where $u_{1} \in U_{1}$, then $u_{1}=0$.

(ii) If $u_{0} \in U_{0}$ and $u_{1} \in U_{1}$ are such that $u_{0} U_{i} u_{1}=u_{1} U_{i} u_{0}=0$, where $i=0$ or $i=1$, then $u_{0} U u_{1}=u_{1} U u_{0}=0$.

(iii) If $u_{1} U_{0}=0$ or $U_{0} u_{1}=0$, where $u_{1} \in U_{1}$, then $u_{1}=0$.

Proof. (i) We have $u_{1} U u_{1} U u_{1} \subseteq u_{1} U u_{1} U_{1} u_{1}+u_{1} U_{1} u_{1} U u_{1}+u_{1} U_{1} u_{1}$ for all $u_{1} \in$ $U_{1}$. Since $u_{1} U_{1} u_{1}=0$, it follows that $u_{1} U u_{1} U u_{1}=0$, and so, since $\mathscr{A}$ is a semiprime algebra, it follows that $u_{1}=0$.

(ii) Assume that $u_{0} U_{0} u_{1}=u_{1} U_{0} u_{0}=0$. Hence $\left(u_{0} U_{1} u_{1}\right) U_{0}\left(u_{0} U_{1} u_{1}\right)=0$. Since $U_{0}$ is a semiprime algebra, we get $u_{0} U_{1} u_{1}=0$. This, together with our assumption, gives $u_{0} U u_{1}=0$.

Now let $u_{0} U_{1} u_{1}=u_{1} U_{1} u_{0}=0$. Hence $\left(u_{0} U_{0} u_{1}\right) U_{1}\left(u_{0} U_{0} u_{1}\right)=0$, and so by (i) we get $u_{0} U_{0} u_{1}=0$. Since, by our assumption, also $u_{0} U_{1} u_{1}=0$, it follows that $u_{0} U u_{1}=0$, as desired.

(iii) Suppose that $u_{1} U_{0}=0$. Hence $u_{1} U_{0} x_{1} u_{1}=0$ and $x_{1} u_{1} U_{0} u_{1}=0$ for all $x_{1} \in \mathscr{A}_{1}$. From (ii) it follows that $u_{1} U x_{1} u_{1}=0$ for all $x_{1} \in A_{1}$. Therefore $x_{1} u_{1} U x_{1} u_{1}=0$, which in turn implies $\mathscr{A}_{1} u_{1}=0$, since $U$ is semiprime. Using (i), we get $u_{1}=0$.

LEMMA 2.3. Let $U$ be a graded ideal of $\mathscr{A}$. Suppose that $a_{1} \in \mathscr{A}_{1}$ is such that $\left[U_{0} a_{1}, U_{0}\right]=$ 0 . Then $U_{0} a_{1} \subseteq Z(\mathscr{A})$.

Proof. We have $0=\left[u_{0} a_{1}, x_{0} v_{0}\right]=\left[u_{0} a_{1}, x_{0}\right] v_{0}+x_{0}\left[u_{0} a_{1}, v_{0}\right]$ for all $u_{0}, v_{0} \in U_{0}$, $x_{0} \in \mathscr{A}_{0}$. Therefore $\left[u_{0} a_{1}, x_{0}\right] U_{0}=0$. Since $\left[u_{0} a_{1}, x_{0}\right] \in U_{1}$, we arrive at $\left[U_{0} a_{1}, \mathcal{A}_{0}\right]=$ 0 by Lemma 2.2(iii). Hence $0=\left[u_{0} a_{1},\left(u_{0} a_{1}\right) x_{1}\right]=u_{0} a_{1}\left[u_{0} a_{1}, x_{1}\right]$ and $0=\left[u_{0} a_{1}\right.$, $\left.x_{1}\left(u_{0} a_{1}\right)\right]=\left[u_{0} a_{1}, x_{1}\right] u_{0} a_{1}$ for all $u_{0} \in U_{0}$ and $x_{1} \in \mathscr{A}_{1}$. Therefore $\left[u_{0} a_{1},\left[u_{0} a_{1}, \mathcal{A}_{1}\right]\right]=$ 0 , which in turn implies $\left[u_{0} a_{1},\left[u_{0} a_{1}, \mathscr{A}\right]\right]=0$ for all $u_{0} \in U_{0}$ and $a_{1} \in \mathscr{A}_{1}$. Since $\mathscr{A}$ is semiprime, the result follows by [8, Lemma 1.1.9]. 
LEMMA 2.4. If $x$ and $y$ are homogeneous elements in $A$ such that $x \perp y$, then $x \circ{ }_{s} D_{i}(y)=0, i=0,1$.

Proof. Since $x \circ_{s} y=0$, it follows that $0=D_{i}\left(x \circ_{s} y\right)=D_{i}(x) \circ_{s} y+(-1)^{|x| i} x \circ_{s} D_{i}(y)$. Using our assumptions and multiplying this identity on the left by $x a, a \in \mathscr{A}$, we arrive at $x a\left(x \circ{ }_{s} D_{i}(y)\right)=0$, which in turn implies $\left(x \circ_{s} D_{i}(y)\right) \mathscr{A}\left(x \circ{ }_{s} D_{i}(y)\right)=0$. Since $\mathscr{A}$ is semiprime, the result follows.

LEMMA 2.5 [6, Lemma 2.6]. It holds that

(i) $\delta_{i}\left(x_{0}, y_{0}\right)=-\delta_{i}\left(y_{0}, x_{0}\right), \delta_{i}\left(x_{0}, y_{1}\right)=-\delta_{i}\left(y_{1}, x_{0}\right)$ and $\delta_{i}\left(x_{1}, y_{1}\right)=\delta_{i}\left(y_{1}, x_{1}\right)$ for all $x_{0}, y_{0} \in A_{0}, x_{1}, y_{1} \in \mathscr{A}_{1}$;

(ii) $D_{i}\left(\left[x_{1}^{2}, y\right]\right)=\left[\left[D_{i}\left(x_{1}\right), x_{1}\right]_{s}, y\right]_{s}+\left[x_{1}^{2}, D_{i}(y)\right]$ for all $x_{1} \in \mathscr{A}_{1}, y \in \mathscr{A}$.

The next lemma is also just a slight extension of [6, Lemma 2.6].

LEMMA 2.6. Let $U$ be a graded ideal of $A$. Suppose that $\delta_{i}\left(U_{0}, \mathscr{A}_{0}\right)=0$. Then

(i) $\left[\delta_{i}\left(\mathscr{A}_{1}, \mathscr{A}_{1}\right), U_{0}\right]=0$;

(ii) $\delta_{i}\left(u_{0}, x_{1}\right) y_{1}=u_{0} \delta_{i}\left(x_{1}, y_{1}\right)-\delta_{i}\left(u_{0} x_{1}, y_{1}\right)$ for all $u_{0} \in U_{0}, x_{1}, y_{1} \in \mathscr{A}_{1}$;

(iii) $\delta_{i}\left(x_{0}, u_{1}\right) y_{1}=x_{0} \delta_{i}\left(u_{1}, y_{1}\right)-\delta_{i}\left(x_{0} u_{1}, y_{1}\right)$ for all $x_{0} \in \mathscr{A}_{0}, u_{1} \in U_{1}$, and $y_{1} \in \mathscr{A}_{1}$;

(iv) $(-1)^{i} y_{1} \delta_{i}\left(u_{0}, x_{1}\right)=\delta_{i}\left(x_{1} u_{0}, y_{1}\right)-\delta_{i}\left(x_{1}, y_{1}\right) u_{0}$ for all $u_{0} \in U_{0}, x_{1} \in \mathscr{A}_{1}$, and $y_{1} \in \mathscr{A}_{1}$

(v) $(-1)^{i} y_{1} \delta_{i}\left(x_{0}, u_{1}\right)=\delta_{i}\left(u_{1} x_{0}, y_{1}\right)-\delta_{i}\left(u_{1}, y_{1}\right) x_{0}$ for all $x_{0} \in A_{0}, u_{1} \in U_{1}$, and $y_{1} \in \mathscr{A}_{1}$

(vi) $\delta_{0}\left(U_{0}, \mathscr{A}_{1}\right) \perp\left[\mathscr{A}_{0}, U_{0}\right]$;

(vii) $\delta_{1}\left(U_{0}, \mathscr{A}_{1}\right) \mathscr{A}_{1}\left[\mathscr{A}_{0}, U_{0}\right]=\left[\mathscr{A}_{0}, U_{0}\right] \mathscr{A}_{1} \delta_{1}\left(U_{0}, \mathscr{A}_{1}\right)=0$.

Proof. We have $D_{i}\left(\left[x_{1}^{2}, u_{0}\right]\right)=\left[D_{i}\left(x_{1}^{2}\right), u_{0}\right]+\left[x_{1}^{2}, D_{i}\left(u_{0}\right)\right]$ for all $x_{1} \in \mathscr{A}_{1}, u_{0} \in U_{0}$. From Lemma 2.5(ii), it follows that $\left[\delta_{i}\left(x_{1}, x_{1}\right), u_{0}\right]=0$ for all $u_{0} \in U_{0}$ and $x_{1} \in \mathscr{A}_{1}$. Linearizing, we get $\left[\delta_{i}\left(\mathscr{A}_{1}, \mathscr{A}_{1}\right), U_{0}\right]=0$.

Now consider the expression $D_{i}\left(u_{0} x_{1} y_{1}\right)$ with $u_{0} \in U_{0}, x_{1}, y_{1} \in \mathscr{A}_{1}$. On the one hand,

$$
\begin{aligned}
D_{i}\left(u_{0}\left(x_{1} y_{1}\right)\right) & =D_{i}\left(u_{0}\right) x_{1} y_{1}+u_{0} D_{i}\left(x_{1} y_{1}\right) \\
& =D_{i}\left(u_{0}\right) x_{1} y_{1}+u_{0}\left(\delta_{i}\left(x_{1}, y_{1}\right)+D_{i}\left(x_{1}\right) y_{1}+(-1)^{i} x_{1} D_{i}\left(y_{1}\right)\right),
\end{aligned}
$$

and on the other hand,

$$
\begin{aligned}
D_{i}\left(\left(u_{0} x_{1}\right) y_{1}\right)= & \delta_{i}\left(u_{0} x_{1}, y_{1}\right)+D_{i}\left(u_{0} x_{1}\right) y_{1}+(-1)^{i} u_{0} x_{1} D_{i}\left(y_{1}\right) \\
= & \delta_{i}\left(u_{0} x_{1}, y_{1}\right)+\left(\delta_{i}\left(u_{0}, x_{1}\right)+D_{i}\left(u_{0}\right) x_{1}+u_{0} D_{i}\left(x_{1}\right)\right) y_{1} \\
& +(-1)^{i} u_{0} x_{1} D_{i}\left(y_{1}\right) .
\end{aligned}
$$

Comparing these two relations, we obtain (ii). In a similar fashion, by computing $D_{i}\left(y_{1} x_{1} u_{0}\right)$ in two different ways (and using Lemma 2.5), we get (iv), by computing $D_{i}\left(x_{0} u_{1} y_{1}\right), x_{0} \in \mathscr{A}_{0}, u_{1} \in U_{1}, y_{1} \in \mathscr{A}_{1}$, we get (iii), and by computing $D_{i}\left(y_{1} u_{1} x_{0}\right)$, we get (v). 
Using (i) and (ii), it follows that $\left[\delta_{i}\left(u_{0}, \mathscr{A}_{1}\right) \mathscr{A}_{1}, u_{0}\right]=0$ for all $u_{0} \in U_{0}$. For any $z_{0} \in \mathscr{A}_{0}$ and $z_{1} \in \mathscr{A}_{1}$, we have $z_{1} z_{0} \in \mathscr{A}_{1}$, and so

$$
\delta_{i}\left(u_{0}, x_{1}\right) z_{1}\left[z_{0}, u_{0}\right]=\left[\delta_{i}\left(u_{0}, x_{1}\right) z_{1} z_{0}, u_{0}\right]-\left[\delta_{i}\left(u_{0}, x_{1}\right) z_{1}, u_{0}\right] z_{0}=0,
$$

proving that

$$
\delta_{i}\left(u_{0}, \mathscr{A}_{1}\right) \mathscr{A}_{1}\left[\mathscr{A}_{0}, u_{0}\right]=0 \quad \forall u_{0} \in U_{0}
$$

Comparing (i) and (iv), we get $\left[\mathscr{A}_{1} \delta_{i}\left(u_{0}, \mathscr{A}_{1}\right), u_{0}\right]=0$, and then, considering an element $z_{0} z_{1} \in \mathscr{A}_{1}$, one obtains

$$
\left[\mathscr{A}_{0}, u_{0}\right] \mathscr{A}_{1} \delta_{i}\left(u_{0}, \mathscr{A}_{1}\right)=0 \quad \forall u_{0} \in U_{0}
$$

A linearization of (2.5) gives $\delta_{i}\left(u_{0}, x_{1}\right) y_{1}\left[z_{0}, v_{0}\right]+\delta_{i}\left(v_{0}, x_{1}\right) y_{1}\left[z_{0}, u_{0}\right]=0$ for all $u_{0}, v_{0} \in U_{0}, z_{0} \in A_{0}, x_{1}, y_{1} \in \mathscr{A}_{1}$. Using (2.6), it follows that

$$
\begin{aligned}
& \left(\delta_{i}\left(u_{0}, x_{1}\right) y_{1}\left[z_{0}, v_{0}\right]\right) a_{1}\left(\delta_{i}\left(u_{0}, x_{1}\right) y_{1}\left[z_{0}, v_{0}\right]\right) \\
& \quad=-\delta_{i}\left(u_{0}, x_{1}\right) y_{1}\left[z_{0}, v_{0}\right] a_{1} \delta_{i}\left(v_{0}, x_{1}\right) y_{1}\left[z_{0}, u_{0}\right]=0
\end{aligned}
$$

for all $a_{1} \in \mathscr{A}_{1}$. Similarly,

$$
\left(\left[z_{0}, v_{0}\right] y_{1} \delta_{i}\left(u_{0}, x_{1}\right)\right) a_{1}\left(\left[z_{0}, v_{0}\right] y_{1} \delta_{i}\left(u_{0}, x_{1}\right)\right)=0 \text {. }
$$

Suppose that $i=0$. Using (2.5) and (2.6), we arrive at $\left[\mathscr{A}_{0}, u_{0}\right] \perp \delta_{0}\left(u_{0}, \mathscr{A}_{1}\right)$ by Lemma 2.2(ii). A linearization of this implies

$$
\begin{aligned}
& \left(\delta_{0}\left(u_{0}, x_{1}\right) y\left[z_{0}, v_{0}\right]\right) a\left(\delta_{0}\left(u_{0}, x_{1}\right) y\left[z_{0}, v_{0}\right]\right) \\
& \quad=-\delta_{0}\left(u_{0}, x_{1}\right) y\left[z_{0}, v_{0}\right] a \delta_{0}\left(v_{0}, x_{1}\right) y\left[z_{0}, u_{0}\right]=0
\end{aligned}
$$

for all $a \in \mathscr{A}$. Therefore $\left[z_{0}, v_{0}\right] \perp \delta_{0}\left(u_{0}, x_{1}\right)$ since $\mathscr{A}$ is semiprime. Assume now that $i=1$. Therefore $\left[z_{0}, v_{0}\right] \mathscr{A}_{1} \delta_{1}\left(u_{0}, x_{1}\right)=0$ and $\delta_{1}\left(u_{0}, x_{1}\right) \mathscr{A}_{1}\left[z_{0}, v_{0}\right]=0$ by (2.7), (2.8), and Lemma 2.2(i). Thereby the proof is completed.

3. Jordan superderivations of degree 0 . By $\left[\mathscr{A}_{0}, \mathscr{A}_{0}\right]$ we will mean the additive subgroup of $\mathscr{A}$ generated by elements of the form $\left[x_{0}, y_{0}\right]$ for all $x_{0}, y_{0} \in \mathscr{A}_{0}$. In what follows, we will denote by $U$ the ideal of $\mathscr{A}$ generated by $\left[\mathscr{A}_{0}, \mathscr{A}_{0}\right]$. Note that $U$ is a graded ideal.

THEOREM 3.1. $\delta_{0}(U, \mathscr{A})=0$.

Proof. Since $D_{0}$ is a Jordan derivation on $\mathscr{A}_{0}$, it follows that

$$
\delta_{0}\left(\mathscr{A}_{0}, \mathscr{A}_{0}\right)=0
$$

by [5]. Therefore we have

$$
\delta_{0}\left(\mathscr{A}_{0}, \mathscr{A}_{1}\right) \perp\left[\mathscr{A}_{0}, \mathscr{A}_{0}\right]
$$


by Lemma 2.6(vi). In particular,

$$
\begin{aligned}
& {\left[y_{1} \delta_{0}\left(x_{0}, x_{1}\right), y_{0}\right] z\left[y_{1} \delta_{0}\left(x_{0}, x_{1}\right), y_{0}\right]} \\
& \quad=\left(y_{1} \delta_{0}\left(x_{0}, x_{1}\right) y_{0} z-y_{0} y_{1} \delta_{0}\left(x_{0}, x_{1}\right) z\right)\left[y_{1} \delta_{0}\left(x_{0}, x_{1}\right), y_{0}\right]=0
\end{aligned}
$$

for all $x_{0}, y_{0} \in \mathscr{A}_{0}, x_{1}, y_{1} \in \mathscr{A}_{1}$, and $z \in \mathscr{A}$, since $y_{1} \delta_{0}\left(x_{0}, x_{1}\right) \in \mathscr{A}_{0}$. In view of the semiprimeness of $\mathscr{A}$, we may conclude that $\left[y_{1} \delta_{0}\left(x_{0}, x_{1}\right), y_{0}\right]=0$ for all $y_{0} \in \mathscr{A}_{0}$. Similarly, $\left[\delta_{0}\left(x_{0}, x_{1}\right) y_{1}, y_{0}\right]=0$, and therefore

$$
\mathscr{A}_{1} \delta_{0}\left(\mathscr{A}_{0}, \mathscr{A}_{1}\right), \delta_{0}\left(\mathscr{A}_{0}, \mathscr{A}_{1}\right) \mathscr{A}_{1} \subseteq Z\left(\mathscr{A}_{0}\right) .
$$

By Lemma 2.1 we arrive at

$$
\delta_{0}\left(\mathscr{A}_{0}, \mathscr{A}_{1}\right)^{2} \subseteq Z(\mathscr{A})
$$

Pick any $u_{0} \in U_{0}$. We have $\delta_{0}\left(u_{0}, x_{1}\right) y_{1} \perp v$ for all $v \in U_{0} \cup U_{1}$ and $x_{1}, y_{1} \in \mathscr{A}_{1}$ by (3.2). Using Lemma 2.4, it follows that

$$
\delta_{0}\left(u_{0}, x_{1}\right) y_{1} D_{0}(v)+D_{0}(v) \delta_{0}\left(u_{0}, x_{1}\right) y_{1}=0 .
$$

Replacing $y_{1}$ by $\delta_{0}\left(u_{0}, x_{1}\right)$ and using (3.5), we obtain $\delta_{0}\left(u_{0}, x_{1}\right)^{2} D_{0}(v)=0$ for all $v \in$ $U_{0} \cup U_{1}$. Since

$$
\left(\delta_{0}\left(u_{0}, x_{1}\right) y_{1}\right)^{2}=\delta_{0}\left(u_{0}, x_{1}\right) y_{1}\left(D_{0}\left(u_{0} x_{1}\right)-D_{0}\left(u_{0}\right) x_{1}-u_{0} D_{0}\left(x_{1}\right)\right) y_{1}
$$

for all $x_{1}, y_{1} \in \mathscr{A}_{1}$, we infer that $\delta_{0}\left(u_{0}, x_{1}\right)^{4}=0$. Again, using (3.5), it follows that $\delta_{0}\left(u_{0}, x_{1}\right)^{2}=0$ since $\mathscr{A}$ is semiprime. Let $v \in U_{1}$. Therefore, multiplying (3.6) on the left by $\delta_{0}\left(u_{0}, x_{1}\right) y_{1}$, we get

$$
\begin{aligned}
0 & =\left(\delta_{0}\left(u_{0}, x_{1}\right) y_{1}\right)^{2} D_{0}(v)+\delta_{0}\left(u_{0}, x_{1}\right) y_{1}\left(D_{0}(v) \delta_{0}\left(u_{0}, x_{1}\right)\right) y_{1} \\
& =\left(\delta_{0}\left(u_{0}, x_{1}\right) y_{1}\right)^{2} D_{0}(v)+D_{0}(v) \delta_{0}\left(u_{0}, x_{1}\right)^{2} y_{1}^{2} \\
& =\left(\delta_{0}\left(u_{0}, x_{1}\right) y_{1}\right)^{2} D_{0}(v)
\end{aligned}
$$

If $v \in U_{0}$, then $\delta_{0}\left(u_{0}, x_{1}\right) y_{1} D_{0}(v)=0$ by (3.6). Using (3.7), we arrive at $\left(\delta_{0}\left(u_{0}, x_{1}\right) y_{1}\right)^{3}=$ 0 , which yields $\delta_{0}\left(u_{0}, x_{1}\right) y_{1}=0$ for all $u_{0} \in U_{0}, x_{1}, y_{1} \in \mathscr{A}_{1}$ by the semiprimeness of $\mathscr{A}_{0}$ and (3.4). Using Lemma 2.2(i), it follows that $\delta_{0}\left(u_{0}, x_{1}\right)=0$ for all $u_{0} \in U_{0}$ and $x_{1} \in \mathscr{A}_{1}$. Analogously, we can show that $\delta_{0}\left(x_{0}, u_{1}\right)=0$ for all $x_{0} \in \mathscr{A}_{0}$ and $u_{1} \in U_{1}$. Therefore

$$
\delta_{0}\left(U_{0}, \mathscr{A}_{1}\right)=\delta_{0}\left(U_{1}, \mathscr{A}_{0}\right)=0 .
$$

Pick any $u_{1} \in U_{1}$ and $x_{1} \in \mathscr{A}_{1}$. Since $x_{1}^{2} \in \mathscr{A}_{0}$, it follows from (3.9) that $\delta_{0}\left(x_{1}^{2}, u_{1}\right)=0$. Hence

$$
D_{0}\left(\left[x_{1}^{2}, u_{1}\right]\right)=\left[D_{0}\left(x_{1}^{2}\right), u_{1}\right]+\left[x_{1}^{2}, D_{0}\left(u_{1}\right)\right] .
$$

On the other hand,

$$
D_{0}\left(\left[x_{1}^{2}, u_{1}\right]\right)=\left[D_{0}\left(x_{1}\right) x_{1}+x_{1} D_{0}\left(x_{1}\right), u_{1}\right]+\left[x_{1}^{2}, D_{0}\left(u_{1}\right)\right]
$$


by Lemma 2.5(ii). Comparing both identities, we arrive at $\left[\delta_{0}\left(x_{1}, x_{1}\right), u_{1}\right]=0$ for all $u_{1} \in$ $U_{1}$ and $x_{1} \in \mathscr{A}_{1}$. Lemma 2.6(i) implies $\left[\delta_{0}\left(x_{1}, x_{1}\right), U\right]=0$ for all $x_{1} \in \mathscr{A}_{1}$. A linearization of this expression gives

$$
\left[\delta_{0}\left(\mathscr{A}_{1}, \mathscr{A}_{1}\right), U\right]=0
$$

by Lemma 2.5(i). From (3.4) and Lemma 2.6(i) and (ii), we get $\left[x_{0} \delta_{0}\left(x_{1}, y_{1}\right), y_{0}\right]=0$ for all $x_{0}, y_{0} \in \mathscr{A}_{0}$ and $x_{1}, y_{1} \in \mathscr{A}_{1}$, which in turn implies

$$
\left[\mathscr{A}_{0}, \mathscr{A}_{0}\right] \delta_{0}\left(\mathscr{A}_{1}, \mathscr{A}_{1}\right)=0 .
$$

Hence $a_{1}\left[x_{0}, y_{0}\right] \delta_{0}\left(x_{1}, y_{1}\right)=0$ for all $a_{1} \in \mathscr{A}_{1}$ by (3.13). Using $a_{1}\left[x_{0}, y_{0}\right] \in U_{1}$ and (3.12), it follows that $\delta_{0}\left(\mathscr{A}_{1}, \mathscr{A}_{1}\right) \mathscr{A}_{1}\left[\mathscr{A}_{0}, \mathscr{A}_{0}\right]=0$. Again, using Lemma 2.6(i) and (3.13), it follows that $\delta_{0}\left(\mathscr{A}_{1}, \mathscr{A}_{1}\right) \mathscr{A}_{0}\left[\mathscr{A}_{0}, \mathscr{A}_{0}\right]=0$. Thus

$$
\left[\mathscr{A}_{0}, \mathscr{A}_{0}\right] \perp \delta_{0}\left(\mathscr{A}_{1}, \mathscr{A}_{1}\right) .
$$

Pick any $u_{1} \in U_{1}$. According to (3.14), we have $\delta_{0}\left(u_{1}, x_{1}\right) \perp v$ for all $v \in U_{0} \cup U_{1}$ and $x_{1} \in A_{1}$. Whence $\delta_{0}\left(u_{1}, x_{1}\right) D_{0}\left(\delta_{0}\left(u_{1}, x_{1}\right) v\right)=0$. By (3.1) and (3.9), we obtain $\delta_{0}\left(u_{1}\right.$, $\left.x_{1}\right)^{2} D_{0}(v)=0$ since $\delta_{0}\left(u_{1}, x_{1}\right) D_{0}\left(\delta_{0}\left(u_{1}, x_{1}\right)\right) v=0$ by (3.14). Obviously,

$$
\delta_{0}\left(u_{1}, x_{1}\right)^{3}=\delta_{0}\left(u_{1}, x_{1}\right)^{2}\left(D_{0}\left(u_{1} x_{1}\right)-D_{0}\left(u_{1}\right) x_{1}-u_{1} D_{0}\left(x_{1}\right)\right)=0 .
$$

Using that $\mathscr{A}_{0}$ is semiprime and $\delta_{0}\left(u_{1}, x_{1}\right) \in Z\left(\mathscr{A}_{0}\right)$, we get $\delta_{0}\left(u_{1}, x_{1}\right)=0$ for all $u_{1} \in U_{1}$ and $x_{1} \in \mathscr{A}_{1}$. Hence

$$
\delta_{0}\left(U_{1}, \mathscr{A}_{1}\right)=0
$$

Thereby the proof is completed.

\section{Jordan superderivations of degree 1}

LEMMA 4.1. Let $D: \mathscr{A}_{0} \rightarrow \mathscr{A}_{1}$ be a linear map satisfying $D(x \circ y)=D(x) \circ y+x \circ D(y)$ for all $x, y \in \mathscr{A}_{0}$. Then, for all $x, y, z, w \in \mathscr{A}_{0}$,

$$
[x, y] \perp(D(z w)-D(z) w-z D(w)) .
$$

Proof. Using [6, Lemma 2.7] (with $\mathscr{A}_{0}=B$ and $M=\mathscr{A}_{1}$ ), it follows that

$$
\begin{aligned}
& {[x, y] \mathscr{A}_{0}[x, y] \mathscr{A}_{0}(D(x y)-D(x) y-x D(y))=0} \\
& (D(x y)-D(x) y-x D(y)) \mathscr{A}_{0}[x, y] \mathscr{A}_{0}[x, y]=0
\end{aligned}
$$

for all $x, y \in \mathscr{A}_{0}$. Write $c=[x, y]$ and $m=D(x y)-D(x) y-x D(y)$ for brevity. Therefore $\left(c x_{0} m\right) x_{1}\left(c x_{0} m\right)=0$ for all $x_{0} \in A_{0}$ and $x_{1} \in \mathscr{A}_{1}$. From Lemma 2.2(i) it follows that $c A_{0} m=0$. Analogously, $m \mathscr{A}_{0} c=0$. Lemma 2.2(ii) implies $m \perp c$. Using [4, Lemma 1.2], the result follows.

LEMMA 4.2. If $a_{1} \in \mathscr{A}_{1}$ is such that $U_{0} a_{1} \subseteq Z(\mathscr{A})$ and $a_{1}\left[U_{0}, U_{0}\right]=0$, then $a_{1} U=0$. 
Proof. We have $0=x\left(u_{0} a_{1}\right)\left[v_{0}, w_{0}\right]=u_{0} a_{1} x\left[v_{0}, w_{0}\right]$ for all $u_{0}, v_{0}, w_{0} \in U_{0}$, and $x \in \mathscr{A}$. It follows that $U_{0} a_{1} \mathscr{A}\left[U_{0}, U_{0}\right]=0$, which in turn implies $a_{1} \mathscr{A}\left[U_{0}, U_{0}\right]=0$ by Lemma 2.2(iii) and the semiprimeness of $U_{0}$. Therefore $0=a_{1} y\left[u_{0}, x_{0} v_{0}\right]=$ $a_{1} y\left[u_{0}, x_{0}\right] v_{0}$ for all $u_{0}, v_{0} \in U_{0}, x_{0} \in \mathscr{A}_{0}$, and $y \in \mathscr{A}$. Hence $a_{1} \mathscr{A}\left[\mathscr{A}_{0}, U_{0}\right]=0$ by Lemma 2.2(iii) and the semiprimeness of $U_{0}$. In the same way, we can show that $a_{1} \mathscr{A}\left[\mathscr{A}_{0}, \mathscr{A}_{0}\right] U_{0}=0$. Again, using Lemma 2.2(iii) and the semiprimeness of $U_{0}$, it follows that $a_{1} \mathscr{A}\left[\mathscr{A}_{0}, \mathscr{A}_{0}\right]=0$ since $a_{1} \mathscr{A}\left[\mathscr{A}_{0}, \mathscr{A}_{0}\right] \subseteq U$. Therefore $a_{1} U=0$.

LEMMA 4.3. Let $a_{0}, b_{0} \in \mathscr{A}_{0}$ be such that $a_{0}\left[\mathscr{A}_{0}, b_{0}\right]=0$. Then $\left[a_{0}, b_{0}\right]=0$.

Proof. We have $0=a_{0}\left[x_{0} y_{0}, b_{0}\right]=a_{0} x_{0}\left[y_{0}, b_{0}\right]$ for all $x_{0}, y_{0} \in A_{0}$. Hence $\left[a_{0}, b_{0}\right] \AA_{0}\left[a_{0}, b_{0}\right]=0$, which yields $\left[a_{0}, b_{0}\right]=0$.

THEOREM 4.4. $\delta_{1}(U, \mathscr{A})=0$.

Proof. By Lemma 4.1, we have $\left[\mathscr{A}_{0}, \mathscr{A}_{0}\right] \perp \delta_{1}\left(\mathscr{A}_{0}, \mathscr{A}_{0}\right)$. In particular, $\left[y_{1} \delta_{1}\left(x_{0}, y_{0}\right)\right.$, $\left.z_{0}\right] \mathscr{A}\left[y_{1} \delta_{1}\left(x_{0}, y_{0}\right), z_{0}\right]=0$ for all $x_{0}, y_{0}, z_{0} \in \mathscr{A}_{0}$ and $y_{1} \in \mathscr{A}_{1}$, since $y_{1} \delta_{1}\left(x_{0}, y_{0}\right) \in$ $\mathscr{A}_{0}$. Using that $\mathscr{A}$ is semiprime, it follows that $\left[y_{1} \delta_{1}\left(x_{0}, y_{0}\right), z_{0}\right]=0$. Analogously, $\left[\delta_{1}\left(x_{0}, y_{0}\right) y_{1}, z_{0}\right]=0$. Hence

$$
\mathscr{A}_{1} \delta_{1}\left(\mathscr{A}_{0}, \mathscr{A}_{0}\right), \delta_{1}\left(\mathscr{A}_{0}, \mathscr{A}_{0}\right) \mathscr{A}_{1} \subseteq Z\left(\mathscr{A}_{0}\right)
$$

Using Lemma 2.1, we infer that

$$
\delta_{1}\left(\mathscr{A}_{0}, \mathscr{A}_{0}\right)^{2} \subseteq Z(\mathscr{A})
$$

Let $u_{0}, v_{0} \in U_{0}$. Then we have $\delta_{1}\left(u_{0}, y_{0}\right) y_{1} \perp v_{0}$ for all $y_{0} \in \mathscr{A}_{0}$ and $y_{1} \in \mathscr{A}_{0}$ by Lemma 4.1. Hence

$$
\delta_{1}\left(u_{0}, y_{0}\right) y_{1} D_{1}\left(v_{0}\right)+D_{1}\left(v_{0}\right) \delta_{1}\left(u_{0}, y_{0}\right) y_{1}=0
$$

by Lemma 2.4. Replacing $y_{1}$ by $\delta_{1}\left(u_{0}, y_{0}\right)$ and using (4.4), it follows that $\delta_{1}\left(u_{0}\right.$, $\left.y_{0}\right)^{2} D_{1}\left(v_{0}\right)=0$ for all $v_{0} \in U_{0}$. Since

$$
\left(\delta_{1}\left(u_{0}, y_{0}\right) y_{1}\right)^{2}=\delta_{1}\left(u_{0}, y_{0}\right) y_{1}\left(D_{1}\left(u_{0} y_{0}\right)-D_{1}\left(u_{0}\right) y_{0}-u_{0} D_{1}\left(y_{0}\right)\right) y_{1}
$$

for all $y_{0} \in \mathscr{A}_{0}, y_{1} \in \mathscr{A}_{1}$, we obtain $\delta_{1}\left(u_{0}, y_{0}\right)^{4}=0$. Using that $\mathscr{A}$ is semiprime and (4.4), we arrive at $\delta_{1}\left(u_{0}, y_{0}\right)^{2}=0$ for all $u_{0} \in U_{0}$ and $y_{0} \in \mathscr{A}_{0}$. Multiplying (4.5) on the left by $\delta_{1}\left(u_{0}, y_{0}\right) y_{1}$, we get

$$
\begin{aligned}
0 & =\left(\delta_{1}\left(u_{0}, y_{0}\right) y_{1}\right)^{2} D_{1}\left(v_{0}\right)+\delta_{1}\left(u_{0}, y_{0}\right) y_{1}\left(D_{1}\left(v_{0}\right) \delta_{1}\left(u_{0}, y_{0}\right)\right) y_{1} \\
& =\left(\delta_{1}\left(u_{0}, y_{0}\right) y_{1}\right)^{2} D_{1}\left(v_{0}\right)+D_{1}\left(v_{0}\right) \delta_{1}\left(u_{0}, y_{0}\right)^{2} y_{1}^{2} \\
& =\left(\delta_{1}\left(w_{0}, y_{0}\right) y_{1}\right)^{2} D_{1}\left(v_{0}\right)
\end{aligned}
$$


Therefore $\left(\delta_{1}\left(u_{0}, y_{0}\right) y_{1}\right)^{3}=0$ by (4.6). The semiprimeness of $\mathcal{A}$, together with (4.3), gives $\delta_{1}\left(u_{0}, y_{0}\right) y_{1}=0$ for all $y_{1} \in \mathscr{A}$. Using Lemma 2.2(i), we obtain

$$
\delta_{1}\left(U_{0}, \mathscr{A}_{0}\right)=0
$$

Using Lemma 2.6(vii), it follows that

$$
\begin{aligned}
0 & =\delta_{1}\left(u_{0}, x_{1}\right) y_{1}\left[x_{0}, y_{0} v_{0}\right] \\
& =\delta_{1}\left(u_{0}, x_{1}\right) y_{1} y_{0}\left[x_{0}, v_{0}\right]+\delta_{1}\left(u_{0}, x_{1}\right) y_{1}\left[x_{0}, y_{0}\right] v_{0}
\end{aligned}
$$

for all $u_{0}, v_{0} \in U_{0}, x_{0}, y_{0} \in \mathscr{A}_{0}$, and $x_{1}, y_{1} \in \mathscr{A}_{1}$, which in turn implies $\delta_{1}\left(u_{0}, x_{1}\right) y_{1}\left[x_{0}\right.$, $\left.y_{0}\right] U_{0}=0$ and analogously $U_{0}\left[x_{0}, y_{0}\right] y_{1} \delta_{1}\left(u_{0}, x_{1}\right)=0$. Since $\delta_{1}\left(u_{0}, x_{1}\right) y_{1}\left[y_{0}, x_{0}\right]$, $\left[y_{0}, x_{0}\right] y_{1} \delta_{1}\left(u_{0}, x_{1}\right) \in U_{1}$, it follows that

$$
\delta_{1}\left(U_{0}, \mathscr{A}_{1}\right) \mathscr{A}_{1}\left[\mathscr{A}_{0}, \mathscr{A}_{0}\right]=\left[\mathscr{A}_{0}, \mathscr{A}_{0}\right] \mathscr{A}_{1} \delta_{1}\left(U_{0}, \mathscr{A}_{1}\right)=0
$$

by Lemma 2.2(iii). Using Lemma 2.6(i), (ii), and (iv), it follows that $\delta_{1}\left(u_{0}, x_{1}\right) y_{1}-$ $y_{1} \delta_{1}\left(u_{0}, x_{1}\right)=\delta_{1}\left(x_{1} u_{0}, y_{1}\right)-\delta_{1}\left(u_{0} x_{1}, y_{1}\right)$ for all $u_{0} \in U_{0}, x_{1}, y_{1} \in \mathscr{A}_{1}$, which yields

$$
\left[\delta_{1}\left(u_{0}, x_{1}\right) y_{1}, v_{0}\right]=\left[y_{1} \delta_{1}\left(u_{0}, x_{1}\right), v_{0}\right], \quad u_{0}, v_{0} \in U_{0}, x_{1}, y_{1} \in \mathscr{A}_{1}
$$

Multiplying this expression on the right by $z_{1}\left[z_{0}, y_{0}\right]$, where $z_{0}, y_{0} \in \mathscr{A}_{0}$ and $z_{1} \in \mathscr{A}_{1}$, we obtain

$$
\left[\delta_{1}\left(U_{0}, \mathscr{A}_{1}\right) \mathscr{A}_{1}, U_{0}\right] \mathscr{A}_{1}\left[\mathscr{A}_{0}, \mathscr{A}_{0}\right]=\left[\mathscr{A}_{0}, \mathscr{A}_{0}\right] \mathscr{A}_{1}\left[\delta_{1}\left(U_{0}, \mathscr{A}_{1}\right) \mathscr{A}_{1}, U_{0}\right]=0
$$

by (4.10). Lemma 2.2(ii) implies

$$
\left[\delta_{1}\left(U_{0}, \mathscr{A}_{1}\right) \mathscr{A}_{1}, U_{0}\right] \perp\left[\mathscr{A}_{0}, \mathscr{A}_{0}\right] .
$$

In particular,

$$
\left[\delta_{1}\left(U_{0}, \mathscr{A}_{1}\right) \mathscr{A}_{1}, U_{0}\right] \mathscr{A}\left[\delta_{1}\left(U_{0}, \mathscr{A}_{1}\right) \mathscr{A}_{1}, U_{0}\right]=0 .
$$

Since $\mathscr{A}$ is semiprime, it follows that $\left[\delta_{1}\left(U_{0}, \mathscr{A}_{1}\right) \mathscr{A}_{1}, U_{0}\right]=0$. From Lemma 2.6(ii) and (iv), we get

$$
\left[U_{0} \delta_{1}\left(\mathscr{A}_{1}, \mathscr{A}_{1}\right), U_{0}\right]=\left[\delta_{1}\left(\mathscr{A}_{1}, \mathscr{A}_{1}\right) U_{0}, U_{0}\right]=0
$$

Using Lemma 2.3, we arrive at

$$
U_{0} \delta_{1}\left(\mathscr{A}_{1}, \mathscr{A}_{1}\right) \subseteq Z(\mathscr{A})
$$

Using (4.15) and Lemma 2.6(i), we obtain $0=\left[u_{0} x_{1}, v_{0}\right]=u_{0}\left[x_{1}, v_{0}\right]+\left[u_{0}, v_{0}\right] x_{1}$ for all $u_{0}, v_{0} \in U_{0}$ and $x_{1} \in \delta_{1}\left(\mathscr{A}_{1}, \mathscr{A}_{1}\right)$. Therefore $\left[U_{0}, U_{0}\right] \delta_{1}\left(\mathscr{A}_{1}, \mathscr{A}_{1}\right)=0$, and similarly $\delta_{1}\left(\mathscr{A}_{1}, \mathscr{A}_{1}\right)\left[U_{0}, U_{0}\right]=0$. Using Lemma 4.2 , it follows that

$$
\delta_{1}\left(\mathscr{A}_{1}, \mathscr{A}_{1}\right) \perp\left[\mathscr{A}_{0}, \mathscr{A}_{0}\right] .
$$


In particular,

$$
\left[\delta_{1}\left(\mathscr{A}_{1}, \mathscr{A}_{1}\right) \mathscr{A}_{1}, \mathscr{A}_{0}\right] \mathscr{A}\left[\delta_{1}\left(\mathscr{A}_{1}, \mathscr{A}_{1}\right) \mathscr{A}_{1}, \mathscr{A}_{0}\right]=0
$$

which in turn implies

$$
\delta_{1}\left(\mathscr{A}_{1}, \mathscr{A}_{1}\right) \mathscr{A}_{1}, \mathscr{A}_{1} \delta_{1}\left(\mathscr{A}_{1}, \mathscr{A}_{1}\right) \subseteq Z\left(\mathscr{A}_{0}\right)
$$

since $\mathscr{A}$ is semiprime. Lemma 2.1 implies

$$
\delta_{1}\left(\mathscr{A}_{1}, \mathscr{A}_{1}\right)^{2} \subseteq Z(\mathscr{A}) .
$$

Pick $u_{1} \in U_{1}, v \in U_{0} \cup U_{1}$, and $x_{1}, y_{1} \in \mathscr{A}_{1}$. We have $\delta_{1}\left(u_{1}, x_{1}\right) y_{1} \perp v$ by (4.17). Using Lemma 2.4, it follows that

$$
\delta_{1}\left(u_{1}, x_{1}\right) y_{1} D_{1}(v)+D_{1}(v) \delta_{1}\left(u_{1}, x_{1}\right) y_{1}=0
$$

Replace $y_{1}$ by $\delta_{1}\left(u_{1}, x_{1}\right)$. Therefore $\delta_{1}\left(u_{1}, x_{1}\right)^{2} D_{1}(v)=0$ for all $v \in U_{0} \cup U_{1}$ by (4.20). Since

$$
\left(\delta_{1}\left(u_{1}, x_{1}\right) y_{1}\right)^{2}=\delta_{1}\left(u_{1}, x_{1}\right) y_{1}\left(D_{1}\left(u_{1} x_{1}\right)-D_{1}\left(u_{1}\right) x_{1}+u_{1} D_{1}\left(x_{1}\right)\right) y_{1}
$$

for all $y_{1} \in \mathscr{A}_{1}$, we get $\delta_{1}\left(u_{1}, x_{1}\right)^{4}=0$. The semiprimeness of $\mathscr{A}$ yields $\delta_{1}\left(u_{1}, x_{1}\right)^{2}=0$ for all $u_{1} \in U_{1}, x_{1} \in \mathscr{A}_{1}$. Let $v \in U_{0}$. Multiplying (4.21) on the left by $\delta_{1}\left(u_{1}, x_{1}\right) y_{1}$, we see that

$$
\begin{aligned}
0 & =\left(\delta_{1}\left(u_{1}, x_{1}\right) y_{1}\right)^{2} D_{1}(v)+\delta_{1}\left(u_{1}, x_{1}\right) y_{1}\left(D_{1}(v) \delta_{1}\left(u_{1}, x_{1}\right)\right) y_{1} \\
& =\left(\delta_{1}\left(u_{1}, x_{1}\right) y_{1}\right)^{2} D_{1}(v)+D_{1}(v) \delta_{1}\left(u_{1}, x_{1}\right)^{2} y_{1}^{2} \\
& =\left(\delta_{1}\left(u_{1}, x_{1}\right) y_{1}\right)^{2} D_{1}(v) .
\end{aligned}
$$

If $v \in U_{1}$, then $\delta_{1}\left(u_{1}, x_{1}\right) y_{1} D_{1}(v)=0$ by (4.21). Therefore $\left(\delta_{1}\left(u_{1}, x_{1}\right) y_{1}\right)^{3}=0$ by (4.22). Since $\mathscr{A}_{0}$ is semiprime and (4.19) holds, we obtain $\delta_{1}\left(u_{1}, x_{1}\right) y_{1}=0$ for all $x_{1}, y_{1} \in \mathscr{A}_{1}$ and $u_{1} \in U_{1}$. By Lemma 2.2(i), it follows that

$$
\delta_{1}\left(U_{1}, \mathscr{A}_{1}\right)=0
$$

Using Lemma 2.6(iii), we arrive at $\delta_{1}\left(\mathscr{A}_{0}, U_{1}\right) \mathscr{A}_{1}=0$, which in turn implies

$$
\delta_{1}\left(\mathscr{A}_{0}, U_{1}\right) \perp \mathscr{A}_{1} .
$$

Therefore

$$
\delta_{1}\left(x_{0}, u_{1}\right) D_{1}\left(x_{1}\right)+D_{1}\left(x_{1}\right) \delta_{1}\left(x_{0}, u_{1}\right)=0
$$

for all $x_{0} \in \mathscr{A}_{0}, x_{1} \in \mathscr{A}_{1}, u_{1} \in U_{1}$ by Lemma 2.4. Again, using Lemma 2.4, we obtain

$$
\delta_{1}\left(x_{0}, u_{1}\right) y_{0} D_{1}\left(x_{1}\right)+D_{1}\left(x_{1}\right) \delta_{1}\left(x_{0}, u_{1}\right) y_{0}=0
$$


for all $y_{0} \in \mathscr{A}_{0}$, since $\delta_{1}\left(x_{0}, u_{1}\right) y_{0} \perp x_{1}$ by (4.25). Multiplying (4.26) on the right by $y_{0} \in$ $\mathscr{A}_{0}$ and comparing the identity so obtained with (4.27), it follows that $\delta_{1}\left(x_{0}, u_{1}\right)\left[y_{0}\right.$, $\left.D_{1}\left(x_{1}\right)\right]=0$ for all $x_{0}, y_{0} \in \mathscr{A}_{0}, u_{1} \in U_{1}$, and $x_{1} \in \mathscr{A}_{1}$. Using Lemma 4.3, we obtain $\left[\delta_{1}\left(x_{0}, u_{1}\right), D_{1}\left(x_{1}\right)\right]=0$. Hence $\left[\delta_{1}\left(x_{0}, u_{1}\right) y_{0}, D_{1}\left(x_{1}\right)\right]=\delta_{1}\left(x_{0}, u_{1}\right)\left[y_{0}, D_{1}\left(x_{1}\right)\right]+$ $\left[\delta_{1}\left(x_{0}, u_{1}\right), D_{1}\left(x_{1}\right)\right] y_{0}=0$ for all $y_{0} \in \mathscr{A}_{0}$. By $(4.27)$, we get $\delta_{1}\left(x_{0}, u_{1}\right) \mathscr{A}_{0} D_{1}\left(x_{1}\right)=0$ for all $x_{0} \in \mathscr{A}_{0}, u_{1} \in U_{1}$, and $x_{1} \in \mathscr{A}_{1}$. Therefore $\delta_{1}\left(x_{0}, u_{1}\right) y_{0} \delta_{1}\left(x_{0}, u_{1}\right)=$ $\delta_{1}\left(x_{0}, u_{1}\right) y_{0}\left(D_{1}\left(x_{0} u_{1}\right)-D_{1}\left(x_{0}\right) u_{1}-x_{0} D_{1}\left(u_{1}\right)\right)=0$ by (4.25). By the semiprimeness of $\mathscr{A}_{0}$, it follows that

$$
\delta_{1}\left(\mathscr{A}_{0}, U_{1}\right)=0
$$

Using Lemma 2.6(ii) and (iv), it follows that $\delta_{1}\left(U_{0}, \mathscr{A}_{1}\right) U_{1}=U_{1} \delta_{1}\left(U_{0}, \mathscr{A}_{1}\right)=0$. Therefore $\delta_{1}\left(U_{0}, \mathscr{A}_{1}\right) \mathscr{A}_{0} U_{1}=0$ and $U_{1} \mathscr{A}_{0} \delta_{1}\left(U_{0}, \mathscr{A}_{1}\right)=0$. By Lemma 2.2(ii), it follows that

$$
\delta_{1}\left(U_{0}, \mathscr{A}_{1}\right) \perp U_{1}
$$

Pick any $u_{0} \in U_{0}$ and $u_{1} \in U_{1}$. Then we have $\delta_{1}\left(u_{0}, x_{1}\right) y_{0} \perp u_{1}$ for all $y_{0} \in \mathscr{A}_{0}$ and $x_{1} \in \mathscr{A}_{1}$. Using (4.28), we get $0=D_{1}\left(\left(\delta_{1}\left(u_{0}, x_{1}\right) y_{0}\right) u_{1}\right)=D_{1}\left(\delta_{1}\left(u_{0}, x_{1}\right) y_{0}\right) u_{1}+\delta_{1}\left(u_{0}\right.$, $\left.x_{1}\right) y_{0} D_{1}\left(u_{1}\right)$. Multiplying this identity on the left by $\delta_{1}\left(u_{0}, x_{1}\right) z_{0}, z_{0} \in \mathscr{A}_{0}$, we obtain $\delta_{1}\left(u_{0}, x_{1}\right) z_{0} \delta_{1}\left(u_{0}, x_{1}\right) y_{0} D_{1}\left(u_{1}\right)=0$. Hence $\delta_{1}\left(u_{0}, x_{1}\right) y_{0} D_{1}\left(u_{1}\right) \mathscr{A}_{0} \delta_{1}\left(u_{0}, x_{1}\right) y_{0} D_{1}\left(u_{1}\right)$ $=0$, which in turn implies

$$
\delta_{1}\left(U_{0}, \mathscr{A}_{1}\right) \mathscr{A}_{0} D_{1}\left(U_{1}\right)=0
$$

since $\mathscr{A}_{0}$ is semiprime. By (4.29), we have $\delta_{1}\left(u_{0}, x_{1}\right) v_{0} \perp y_{1}$ for all $v_{0} \in U_{0}$ and $y_{1} \in \mathscr{A}_{1}$. Whence

$$
\delta_{1}\left(u_{0}, x_{1}\right) v_{0} D_{1}\left(y_{1}\right)+D_{1}\left(y_{1}\right) \delta_{1}\left(u_{0}, x_{1}\right) v_{0}=0
$$

by Lemma 2.4. Since also $x_{0} \delta_{1}\left(u_{0}, x_{1}\right) v_{0} \perp y_{1}, x_{0} \in \mathcal{A}_{0}$, we arrive at

$$
x_{0} \delta_{1}\left(u_{0}, x_{1}\right) v_{0} D_{1}\left(y_{1}\right)+D_{1}\left(y_{1}\right) x_{0} \delta_{1}\left(u_{0}, x_{1}\right) v_{0}=0
$$

for all $u_{0}, v_{0} \in U_{0}, x_{0} \in \mathscr{A}_{0}, x_{1}, y_{1} \in \mathscr{A}_{1}$ by Lemma 2.4. If we multiply (4.31) on the left by $x_{0}$ and compare the identity so obtained with (4.32), it follows that

$$
\left[\mathscr{A}_{0}, D_{1}\left(\mathscr{A}_{1}\right)\right] \delta_{1}\left(U_{0}, \mathscr{A}_{1}\right) U_{0}=0 \text {. }
$$

Since $\left[\mathscr{A}_{0}, D_{1}\left(\mathscr{A}_{1}\right)\right] \delta_{1}\left(U_{0}, \mathscr{A}_{1}\right) \subseteq U_{0}$, we obtain $\left[\mathscr{A}_{0}, D_{1}\left(\mathscr{A}_{1}\right)\right] \delta_{1}\left(U_{0}, \mathscr{A}_{1}\right)=0$ by the semiprimeness of $U_{0}$. Therefore also $\delta_{1}\left(U_{0}, \mathscr{A}_{1}\right)\left[\mathscr{A}_{0}, D_{1}\left(\mathscr{A}_{1}\right)\right]=0$. Using Lemma 4.3 , it follows that $\left[\delta_{1}\left(U_{0}, \mathscr{A}_{1}\right), D_{1}\left(\mathscr{A}_{1}\right)\right]=0$, which yields $\left[\delta_{1}\left(U_{0}, \mathscr{A}_{1}\right) \mathscr{A}_{0}, D_{1}\left(\mathscr{A}_{1}\right)\right]=0$. Using (4.31), we obtain $\delta_{1}\left(U_{0}, \mathscr{A}_{1}\right) U_{0} D_{1}\left(\mathscr{A}_{1}\right)=0$. By (4.29), we arrive at

$$
\delta_{1}\left(u_{0}, x_{1}\right) v_{0} \delta_{1}\left(u_{0}, x_{1}\right)=\delta_{1}\left(u_{0}, x_{1}\right) v_{0}\left(D_{1}\left(u_{0} x_{1}\right)-D_{1}\left(u_{0}\right) x_{1}-u_{0} D_{1}\left(x_{1}\right)\right)=0
$$


for all $v_{0} \in U_{0}$. Using that $U_{0}$ is semiprime, it follows that $U_{0} \delta_{1}\left(U_{0}, \mathscr{A}_{1}\right)=0$, which in turn implies $U \delta_{1}\left(U_{0}, \mathscr{A}_{1}\right)=0$. Analogously, $\delta_{1}\left(U_{0}, \mathscr{A}_{1}\right) U=0$. Using (4.8), we obtain

$$
0=\delta_{1}\left(u_{0}, x_{1}\right) x_{0} D_{1}\left(\left(\delta_{1}\left(u_{0}, x_{1}\right) y_{0}\right) v_{0}\right)=\delta_{1}\left(u_{0}, x_{1}\right) x_{0} \delta_{1}\left(u_{0}, x_{1}\right) y_{0} D_{1}\left(v_{0}\right)
$$

for all $x_{0}, y_{0} \in \mathscr{A}_{0}, u_{0}, v_{0} \in U_{0}$, and $x_{1} \in \mathscr{A}_{1}$. Hence

$$
\left(\delta_{1}\left(u_{0}, x_{1}\right) x_{0} D_{1}\left(v_{0}\right)\right) \mathscr{A}_{1}\left(\delta_{1}\left(u_{0}, x_{1}\right) x_{0} D_{1}\left(v_{0}\right)\right)=0,
$$

which yields $\delta_{1}\left(U_{0}, \mathscr{A}_{1}\right) \mathscr{A}_{0} D_{1}\left(U_{0}\right)=0$ by Lemma 2.2(i). Using (4.30), it follows that

$$
\delta_{1}\left(u_{0}, x_{1}\right) x_{0} \delta_{1}\left(u_{0}, x_{1}\right)=\delta_{1}\left(u_{0}, x_{1}\right) x_{0}\left(D_{1}\left(u_{0} x_{1}\right)-D_{1}\left(u_{0}\right) x_{1}-u_{0} D_{1}\left(x_{1}\right)\right)=0 .
$$

Consequently,

$$
\delta_{1}\left(U_{0}, \mathscr{A}_{1}\right)=0
$$

Thereby the proof is completed.

5. Proof of Theorem 1.1. Theorems 3.1 and 4.4 show that $D(u x)=D_{0}(u x)+$ $D_{1}(u x)=D_{0}(u) x+u D_{0}(x)+D_{1}(u) x+(-1)^{|u|} u D_{1}(x)$. Therefore $D \mid U$ is the sum of superderivations of degrees 0 and 1 .

Set $V=\operatorname{Ann}(U)$. Pick $v_{0} \in V_{0}$. We have

$$
\left[v_{0}, x_{0}\right] y_{0}\left[v_{0}, x_{0}\right]=v_{0}\left(x_{0} y_{0}\left[v_{0}, x_{0}\right]\right)-x_{0} v_{0}\left(y_{0}\left[v_{0}, x_{0}\right]\right)=0
$$

for all $v_{0} \in V_{0}, x_{0}, y_{0} \in \mathscr{A}_{0}$, since $y_{0}\left[v_{0}, x_{0}\right], x_{0} y_{0}\left[v_{0}, x_{0}\right] \in U_{0}$. By the semiprimeness of $\mathscr{A}_{0}$, we arrive at $\left[v_{0}, x_{0}\right]=0$ for all $x_{0} \in \mathscr{A}_{0}$ and $v_{0} \in V_{0}$.

We show that

$$
\delta_{1}\left(\mathscr{A}_{0}, \mathscr{A}_{1}\right) \perp\left[\mathscr{A}_{0}, \mathscr{A}_{0}\right]
$$

Consider the expression $D_{1}\left(u_{0} x_{0} y_{1}\right)$ with $u_{0} \in U_{0}, x_{0} \in \mathscr{A}_{0}, y_{1} \in \mathscr{A}_{1}$. On the one hand,

$$
D_{1}\left(u_{0}\left(x_{0} y_{1}\right)\right)=D_{1}\left(u_{0}\right) x_{0} y_{1}+u_{0} D_{1}\left(x_{0} y_{1}\right)
$$

and, on the other hand,

$$
\begin{aligned}
D_{1}\left(\left(u_{0} x_{0}\right) y_{1}\right) & =D_{1}\left(u_{0} x_{0}\right) y_{1}+u_{0} x_{0} D_{1}\left(y_{1}\right) \\
& =D_{1}\left(u_{0}\right) x_{0} y_{1}+u_{0} D_{1}\left(x_{0}\right) y_{1}+u_{0} x_{0} D_{1}\left(y_{1}\right) .
\end{aligned}
$$

Comparing these two relations, we obtain $U_{0} \delta_{1}\left(\mathscr{A}_{0}, \mathscr{A}_{1}\right)=0$. In particular,

$$
\left[\mathscr{A}_{0}, \mathscr{A}_{0}\right] \mathscr{A}_{0} \delta_{1}\left(\mathscr{A}_{0}, \mathscr{A}_{1}\right)=0
$$


We also have $\mathscr{A}_{1}\left[\mathscr{A}_{0}, \mathscr{A}_{0}\right] \mathscr{A}_{1} \delta_{1}\left(\mathscr{A}_{0}, \mathscr{A}_{1}\right)=0$. By Lemma 2.2(i), we arrive at $\left[\mathscr{A}_{0}\right.$, $\left.\mathscr{A}_{0}\right] \mathscr{A}_{1} \delta_{1}\left(\mathscr{A}_{0}, \mathscr{A}_{1}\right)=0$ and the result follows. If $U=0$, then $\mathscr{A}_{0}$ is commutative. Note that $\delta_{0}(x, y), \delta_{1}(x, y) \in V, x, y \in \mathscr{A}_{0} \cup \mathscr{A}_{1}$, by (3.1), (3.2), (3.14), (4.17), (5.2), and Lemma 4.1. Therefore $V=0$ implies that $D$ is a superderivation.

We have $U V=0$, and hence $U \cap V=0$ since $\mathscr{A}$ is semiprime. Suppose that $(U+V) \cap$ $I=0$ for some graded ideal $I$ of $\mathscr{A}$. Hence $U I=V I=0$. Therefore $I \subseteq \operatorname{Ann}(U) \cap$ $\operatorname{Ann}(V)=\operatorname{Ann}(U) \cap \operatorname{Ann}(\operatorname{Ann}(U))=0$. Thus $U \oplus V$ is an essential ideal of $\mathscr{A}$. The proof is completed.

Acknowledgment. I am thankful to Professor Matej Brešar for help and encouragement.

\section{REFERENCES}

[1] W. E. Baxter and W. S. Martindale III, Jordan homomorphisms of semiprime rings, J. Algebra 56 (1979), no. 2, 457-471.

[2] K. I. Beidar, M. Brešar, and M. A. Chebotar, Jordan superhomomorphisms, Comm. Algebra 31 (2003), no. 2, 633-644.

[3] M. Brešar, Jordan derivations on semiprime rings, Proc. Amer. Math. Soc. 104 (1988), no. 4, 1003-1006.

[4] _ Jordan mappings of semiprime rings, J. Algebra 127 (1989), no. 1, 218-228.

[5] J. M. Cusack, Jordan derivations on rings, Proc. Amer. Math. Soc. 53 (1975), no. 2, 321-324.

[6] M. Fošner, Jordan superderivations, Comm. Algebra 31 (2003), no. 9, 4533-4545.

[7] I. N. Herstein, Jordan derivations of prime rings, Proc. Amer. Math. Soc. 8 (1957), 1104-1110.

[8] _ Rings with Involution, The University of Chicago Press, Illinois, 1976.

[9] F. Montaner, On the Lie structure of associative superalgebras, Comm. Algebra 26 (1998), no. 7, 2337-2349.

Maja Fošner: Institute of Mathematics, Physics, and Mechanics, Jadranska 19, 1000 Ljubljana, Slovenia

E-mail address: maja.fosner@uni-mb.si 


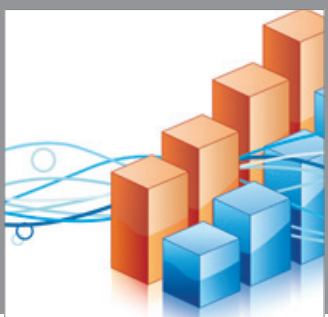

Advances in

Operations Research

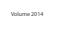

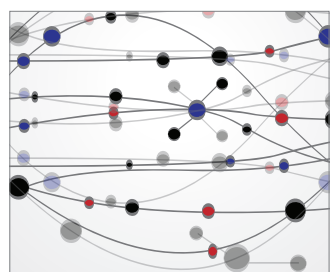

\section{The Scientific} World Journal
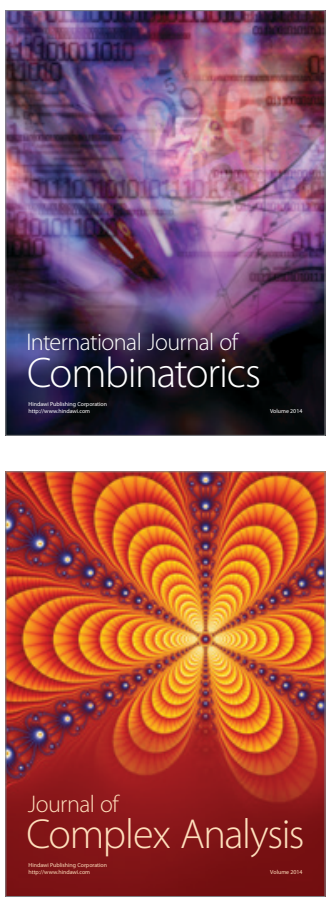

International Journal of

Mathematics and

Mathematical

Sciences
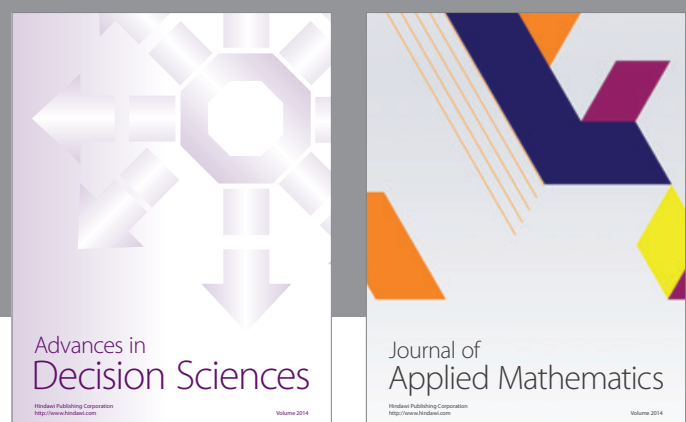

Journal of

Applied Mathematics
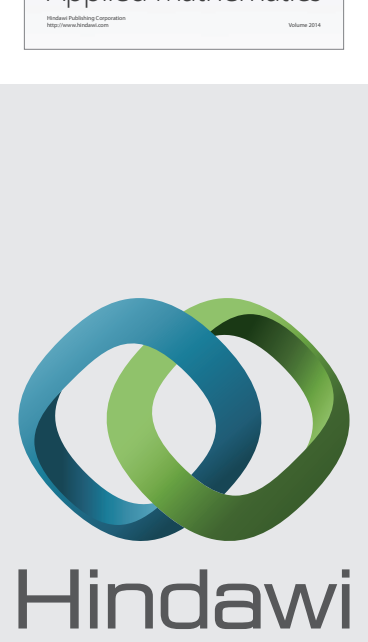

Submit your manuscripts at http://www.hindawi.com
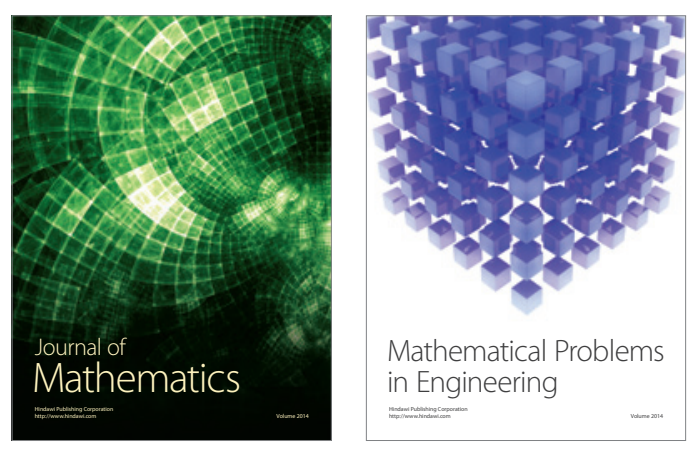

Mathematical Problems in Engineering
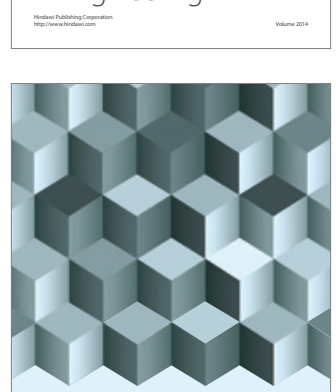

Journal of

Function Spaces
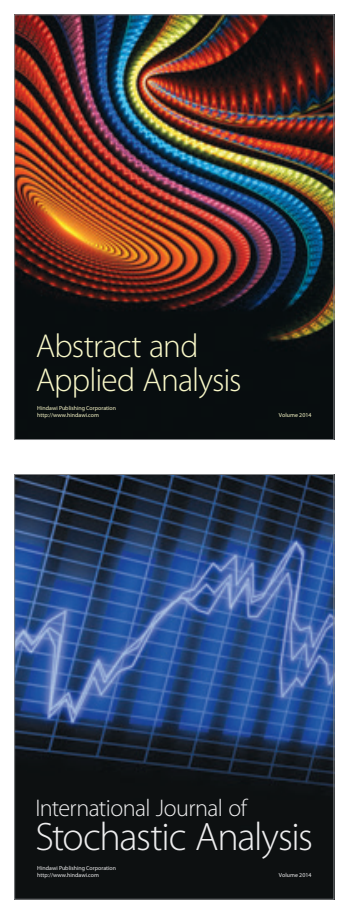

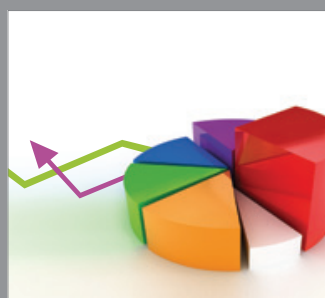

ournal of

Probability and Statistics

Promensencen
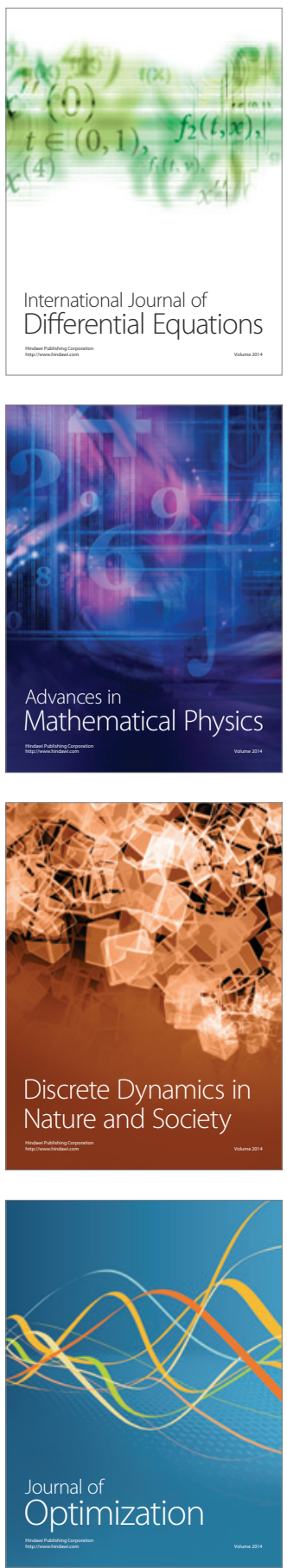